\title{
INTERPRETATION OF $\mathrm{CO}_{2}$ EXHALATION RATE DATA FROM DEMETHYLATION OF AMINOPYRINE AND ITS METABOLITE MONOMETHYLAMINOANTIPYRINE
}

\author{
J.C. RHODES, L.J. AARONS \& J.B. HOUSTON \\ Department of Pharmacy, University of Manchester, Manchester M13 9PL
}

1 Aminopyrine breath tests make use of the commercially available ( $N$-dimethyl- $\left.\left[{ }^{14} \mathrm{C}\right]\right)$-aminopyrine. A pharmacokinetic model has been proposed to relate ${ }^{14} \mathrm{CO}_{2}$ exhalation rates (CER) to the demethylation of $\left(\left[{ }^{14} \mathrm{C}\right]\right.$-methyl)-aminopyrine (AP) and -monomethylaminoantipyrine (MAP).

2 Computer simulations based on the model show that the shape of the CER-time profile is largely dependent on the ratio of the MAP to AP elimination rate constants. If this ratio equals 0.5 then the CERs decline in the monoexponential fashion. Ratios less than 0.5 result in concave biexponential curves whereas ratios greater than 0.5 result in convex curves. When demethylation is not complete for both compounds the transfer from biexponential to monoexponential behaviour will only occur at ratios greater than 0.5 .

3 The resolution of concave biexponential CER-time profiles to give accurate estimates of AP and MAP elimination rate constants can only be achieved when the length of the experiment is adequate. The commonly employed $2 \mu \mathrm{Ci}$ tracer dose of aminopyrine is insufficient to monitor CER over the necessary time period to detect the proposed biexponential decline.

\section{Introduction}

The model compound aminopyrine is extensively metabolised by the hepatic microsomal enzyme system. $N$-demethylation is the major route of metabolism in man (Brodie \& Axelrod, 1950) and other animal species (Roots et al., 1978; Lockwood \& Houston, 1979) and the formaldehyde generated by this reaction is further oxidised to formic acid and finally carbon dioxide (Waydhas et al., 1978). A full description of the fate of formaldehyde has yet to be achieved, however it is known that the rate limiting step in this catenary sequence is the formation of formaldehyde via the demethylation of aminopyrine (Aebi et al., 1963). Lauterburg \& Bircher (1973) proposed that by monitoring ${ }^{14} \mathrm{CO}_{2}$ production after administration of $\left(\mathrm{N}\right.$-dimethyl-[ $\left.\left.{ }^{14} \mathrm{C}\right]\right)$-aminopyrine the demethylation rate for this drug could be measured and hence the activity of the hepatic microsomal enzyme quantified.

The use of aminopyrine ${ }^{-14} \mathrm{CO}_{2}$ exhalation rate (AP-CER) tests has provoked much interest in view of their non-invasive nature. Hepner \& Vesell (1975) developed the aminopyrine breath test where a single sample of exhaled air is collected $2 \mathrm{~h}$ after ingestion of $\left[{ }^{14} \mathrm{C}\right]-\mathrm{AP}$. Good correlations between the AP breath test and the plasma clearance of AP have been reported in patients with liver disease and con- gestive heart failure and in normal subjects (Hepner \& Vesell, 1976; Hepner et al., 1978). In conditions of impaired hepatic enzyme activity this test would appear to be comparable to other liver function tests (Hepner \& Vesell, 1976; Bircher et al., 1976). However as illustrated by Henry et al. (1979), the sensitivity of the AP breath test may be quite limited in conditions of enhanced hepatic enzyme activity. Only when serial samples are obtained and a breath halflife calculated can a true appreciation of the changes in CER-time course be obtained.

One major anomaly of the AP-CER tests is the inequivalence between the half-life of AP in the plasma and the half-life obtained from the CER; the latter being reported to be $20-80 \%$ larger than the former (Bircher et al., 1976; Noordhoek et al., 1978). As pointed out by several investigators the $\left[{ }^{14} \mathrm{C}\right]-\mathrm{AP}$ commercially available is radio-labelled in both methyl groups in the 4-amino position. Thus CERs reflect both the demethylation of the tertiary amine AP to its secondary amine metabolite (MAP) plus the demethylation of the secondary amine to a primary amine metabolite. We have shown that in rats the plasma half-life of MAP is three times longer than the AP half-life (Lockwood \& Houston, 1980) and the CER-time profile measured over a $6 \mathrm{~h}$ period 
displayed a biexponential decline (Houston et al., 1981). Resolution of the two exponentials by curve stripping yields a slow $\beta$ half-life which is comparable to MAP plasma half-life and a fast $\alpha$ half-life which is comparable to AP plasma half-life. In a recent investigation (Roots et al., 1980) guinea pigs were administered $\left[{ }^{14} \mathrm{C}\right]-\mathrm{AP}$ and CER monitored over 4-6 $h$. The biphasic nature of the CER-time plot was less pronounced than in the rat study and consequently the exponentials difficult to resolve. As part of the guinea-pig study $\left[{ }^{14} \mathrm{C}\right]-\mathrm{MAP}$ was also administered. A monoexponential decline in CER was observed but the half-life measured did not equate with the $\beta$-halflife from the $\left[{ }^{14} \mathrm{C}\right]$-AP study.

Hence there is some confusion in the literature concerning the association between AP and MAP metabolism and CER measurements. In an attempt to resolve the situation we have formulated a simple pharmacokinetic model to describe the relationship between CER, AP and MAP plasma concentration data. Using this model we have stimulated CER-time profiles under a number of conditions to investigate which parameters determine the shape of the profile. We believe this approach clarifies certain of the apparently paradoxical reports in the literature.

\section{Methods}

\section{The model}

As shown in scheme 1 , aminopyrine (AP) is demethylated via two consecutive steps to form monomethylaminoantipyrine (MAP) and then aminoantipyrine (AA). The single carbon moiety is lost as formaldehyde, oxidised to yield formic acid and then carbon dioxide which is exhaled. In the model these oxidation steps are considered to occur so rapidly that carbon dioxide exhalation instantaneously follows demethylation.

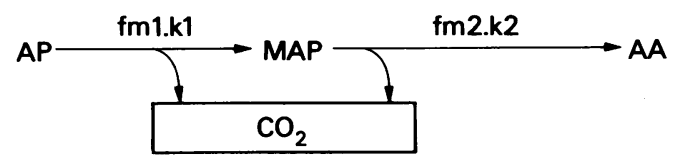

Scheme 1: $\mathrm{CO}_{2}$ production from aminopyrine and its metabolite monomethylaminoantipyrine.

AP is administered intravenously and the first order rate constants $k 1$ and $k 2$ describe the elimination of AP and MAP, respectively, from the body. The fraction of AP which is demethylated is $\mathrm{fm} 1$ and $\mathrm{fm} 2$ is the fraction of MAP demethylated to AA.

The rate of elimination of AP from the body may be described by

$$
\frac{\mathrm{d}(\mathrm{AP})}{\mathrm{dt}}=-\mathrm{k} 1 . \mathrm{AP}
$$

which when integrated becomes

$$
\mathrm{AP}=\mathrm{D} \cdot \mathrm{e}^{-\mathrm{k} 1 . \mathrm{t}}
$$

where $\mathrm{D}$ is the dose of AP administered in moles.

Similarly equations (3) and (4) describe the elimination of MAP following AP administration

$$
\begin{aligned}
\frac{\mathrm{d}(\mathrm{MAP})}{\mathrm{dt}} & =\mathrm{fm} 1 . \mathrm{k} 1 . \mathrm{AP}-\mathrm{k} 2 . \mathrm{MAP} \\
\text { MAP } & =\frac{\mathrm{fm} 1 . \mathrm{k} 1 . \mathrm{D}}{\mathrm{k} 1-\mathrm{k} 2}\left(\mathrm{e}^{-\mathrm{k} 2 . \mathrm{t}}-\mathrm{e}^{-\mathrm{k} 1 . t}\right)
\end{aligned}
$$

where $\mathbf{k} 1 \neq \mathbf{k} 2$.

Therefore the rate for carbon dioxide production from the demethylation of AP and subsequently MAP is

$$
\frac{\mathrm{d}\left(\mathrm{CO}_{2}\right)}{\mathrm{dt}}=\mathrm{fm} 1 . \mathrm{k} 1 . A P+\mathrm{fm} 2 . \mathrm{k} 2 . \mathrm{MAP}
$$

Substitution of equations (2) and (4) into equation (5) yields

$$
\begin{aligned}
\frac{\mathrm{d}\left(\mathrm{CO}_{2}\right)}{\mathrm{dt}} & =\mathrm{fm} 1 \cdot \mathrm{k} 1 \cdot \mathrm{D} \cdot \mathrm{e}^{-\mathrm{k} 1 \cdot \mathrm{t}} \\
& +\frac{\mathrm{fm} 2 \cdot \mathrm{k} 2 \cdot \mathrm{fm} 1 \cdot \mathrm{k} 1 \cdot \mathrm{D}}{\mathrm{k} 1-\mathrm{k} 2}\left(\mathrm{e}^{\left.-\mathrm{k} 2 \cdot \mathrm{t}-\mathrm{e}^{-\mathrm{k} 1 \cdot t}\right)}\right. \\
& =\left(\frac{\mathrm{fm} 1 \cdot \mathrm{k} 1 \cdot \mathrm{D} \cdot[\mathrm{k} 1-(1+\mathrm{fm} 2) \cdot \mathrm{k} 2)]}{\mathrm{k} 1-\mathrm{k} 2}\right) \mathrm{e}^{-\mathrm{k} 1 . t} \\
& +\left(\frac{\mathrm{fm} 1 \cdot \mathrm{k} 1 \cdot \mathrm{D} \cdot \mathrm{fm} 2 \cdot \mathrm{k} 2}{\mathrm{k} 1-\mathrm{k} 2}\right) \mathrm{e}^{-\mathrm{k} 2 . t}
\end{aligned}
$$

Equation 7 may be simplified and on the assumption that $\mathrm{d}\left(\mathrm{CO}_{2}\right) / \mathrm{dt}=\mathrm{CO}_{2}$ exhalation rate $(\mathrm{CER})$, and equation (8) produced

CER =

$\frac{\mathrm{fm} 1 . \mathrm{k} 1 . \mathrm{D}}{\mathrm{k} 1-\mathrm{k} 2}\left([\mathrm{k} 1-(1+\mathrm{fm} 2) \cdot \mathrm{k} 2] \mathrm{e}^{-\mathrm{k} 1 . t}+\mathrm{fm} 2 \cdot \mathrm{k} 2 \cdot \mathrm{e}^{-\mathrm{k} 2 . t}\right)$

For the computer simulations equation (8) was normalised by introducing the terms $\tau(\tau=\mathrm{k} 1 . \mathrm{t}), \mathrm{K}$ $(K=k 2 / k 1)$ and $C E R^{\prime}\left(C E R^{\prime}=C E R / k 1\right.$.D) to give equation (9).

$\mathrm{CER}^{\prime}=\frac{\mathrm{fml}}{1-\mathrm{K}}\left([1-\mathrm{K}(1+\mathrm{fm} 2)] \mathrm{e}^{-\tau}+\mathrm{fm} 2 \cdot \mathrm{K} \cdot \mathrm{e}^{-\mathrm{K} \cdot \tau}\right)$

In its general form equation (9) is

$$
\mathrm{CER}^{\prime}=\mathbf{A} \cdot \mathrm{e}^{-\tau}+\mathbf{B} \cdot \mathrm{e}^{-\mathrm{K} \cdot \tau}
$$

where

$$
A=\frac{f m 1[1-K(1+f m 2)]}{1-K}
$$




$$
B=\frac{\mathrm{fm} 1 \cdot \mathrm{fm} 2 \cdot \mathrm{K}}{1-\mathrm{K}}
$$

A special case exists when $k 1=k 2$. In this situation equation (3) integrates to give

$$
\text { MAP }=\text { fm1.k1.D.t. } e^{-k l . t}
$$

The final equation equivalent to equation (9) for the special case is

$$
\mathrm{CER}^{\prime}=\mathrm{fm} 1(1+\mathrm{fm} 2) \tau \cdot \mathrm{e}^{-\tau}
$$

CER data which conforms to equation (14) rather than equation (9) will not show a terminal linear region when plotted on semi-logarithmic graph paper.

\section{Simulations}

In order to investigate the effect of $\mathrm{K}$ on the shape of the CER-time profile both $\mathrm{fm} 1$ and $\mathrm{fm} 2$ were set to unity. Under this condition equation (9) reduces to

$$
\mathrm{CER}^{\prime}=\frac{1}{1-\mathrm{K}}\left((1-2 \mathrm{~K}) \cdot \mathrm{e}^{-\tau}+\mathrm{K} \cdot \mathrm{e}^{-\mathrm{K} \cdot \tau}\right)
$$

Equation (15) was used to simulate CER-time profiles using an Apple II micro computer for a range of $\mathrm{K}$ values from 0.1 to 5 .

\section{Resolution of biexponential CER data}

A standard procedure for resolving the exponents of biexponential equations such as equation (9) is curve stripping (Gibaldi \& Perrier, 1975). Providing the two exponents differ then a semi-logarithmic plot of CER against mid-point time will decline in a concave fashion with a terminal linear slope governed by the smallest exponent. Extrapolation of this slope back to zero time allows calculation of residuals (observed minus extrapolated) for each time point during the initial phase. Plotting the residuals on a semilogarithmic scale provides a straight line, the slope of which is controlled by the largest exponent. The accuracy of this procedure for CER data was determined for certain values of $\mathrm{K}$ over different experimental time periods $(T)$.

Twenty data points were generated using equation (15) at equal time intervals from zero to $T$ for values of $\mathrm{K}$ between 0.1 and 0.5 . The last four data points were used to describe the terminal slope and the curve was stripped. The percentage error on the estimates of the rate constants was calculated from the difference between the rate constant obtained by the above treatment of data and the theoretical rate constants used to generate the data.

\section{Results and discussion}

The $\mathrm{CO}_{2}$ exhalation rate $\left(\mathrm{CER}^{\prime}\right)$ curves plotted as a function of both $\tau$ and multiples of aminopyrine (AP) half-life are shown in Figure 1. The shape of the CER curve is dependent upon the ratio $K(\mathrm{k} 2 / \mathrm{k} 1)$. A critical value for $\mathrm{K}$ is 0.5 since the biexponential equation (15) condenses to give equation (16) in this situation.

$$
\mathrm{CER}^{\prime}=\mathrm{e}^{-0.5 \tau}
$$

When $\mathrm{K}$ is less than 0.5 , the CER curve is concave (see Figure 1a). Initially both demethylations contribute to the $\mathrm{CO}_{2}$ production and the CER is a hybrid. After some time the first term in equation (15) (representing AP demethylation) will approach zero while the second term (representing MAP demethylation) is still measurable. Thus the terminal phase data points may be used to obtain $\mathbf{k} 2$ and, following curve stripping, the residual points used to obtain $\mathrm{k} 1$.

When $\mathrm{K}$ exceeds 0.5 , the CER is convex (see Figure $1 \mathrm{~b}$ ). This is particularly apparent for $\mathrm{K}$ ratios greater than 1 where the coefficient $A$ (equation 11 ) is positive and coefficient $B$ (equation 12) is negative and the terminal phase determines $k 1$ (curve 5). The signs of the coefficients are reversed for $K$ ratios between 0.5 and 1 and a value of $\mathrm{k} 2$ may be estimated from the terminal phase (curve 4). In the special case where $\mathbf{K}=1$, as outlined previously, no terminal linear phase exists and hence no graphical determination of rate constants is possible.

The simulations in Figure 1 were generated for the case where both $\mathrm{fm} 1$ and $\mathrm{fm} 2$ equal unity. However the shapes of the CER-time profiles for particular K values will not be altered if either or both of these parameters are less than one. These fractions contribute only to the coefficients (equations 11 and 12) not the exponents. Therefore the overall elimination rate constants for AP (k1) and MAP (k2) are still obtainable from the exponential decline. However, as a consequence of reduced coefficients the total area under the CER-time curve will decrease.

Interestingly $\mathrm{fm} 2$ also governs the transfer from biexponential to monoexponential CER behaviour. Inspection of equation (11) reveals that the coefficient $A$ will equal zero when

$$
K=\frac{1}{1+\mathrm{fm} 2}
$$

As described above the critical value of $K$ for $f m 2=1$ will be 0.5 . As fm2 decreases, this critical value for $K$ will increase. However, within the $\mathrm{fm} 2$ range of 0.7 to 1 the critical value for $K$ is relatively constant (between 0.5 and 0.6).

Both parameters, $K$ and $\mathrm{fm} 2$, will determine whether the concave nature of the CER-time profile is apparent. Accurate estimates of the exponents will only be achieved if the length of experiment $(T)$ is 


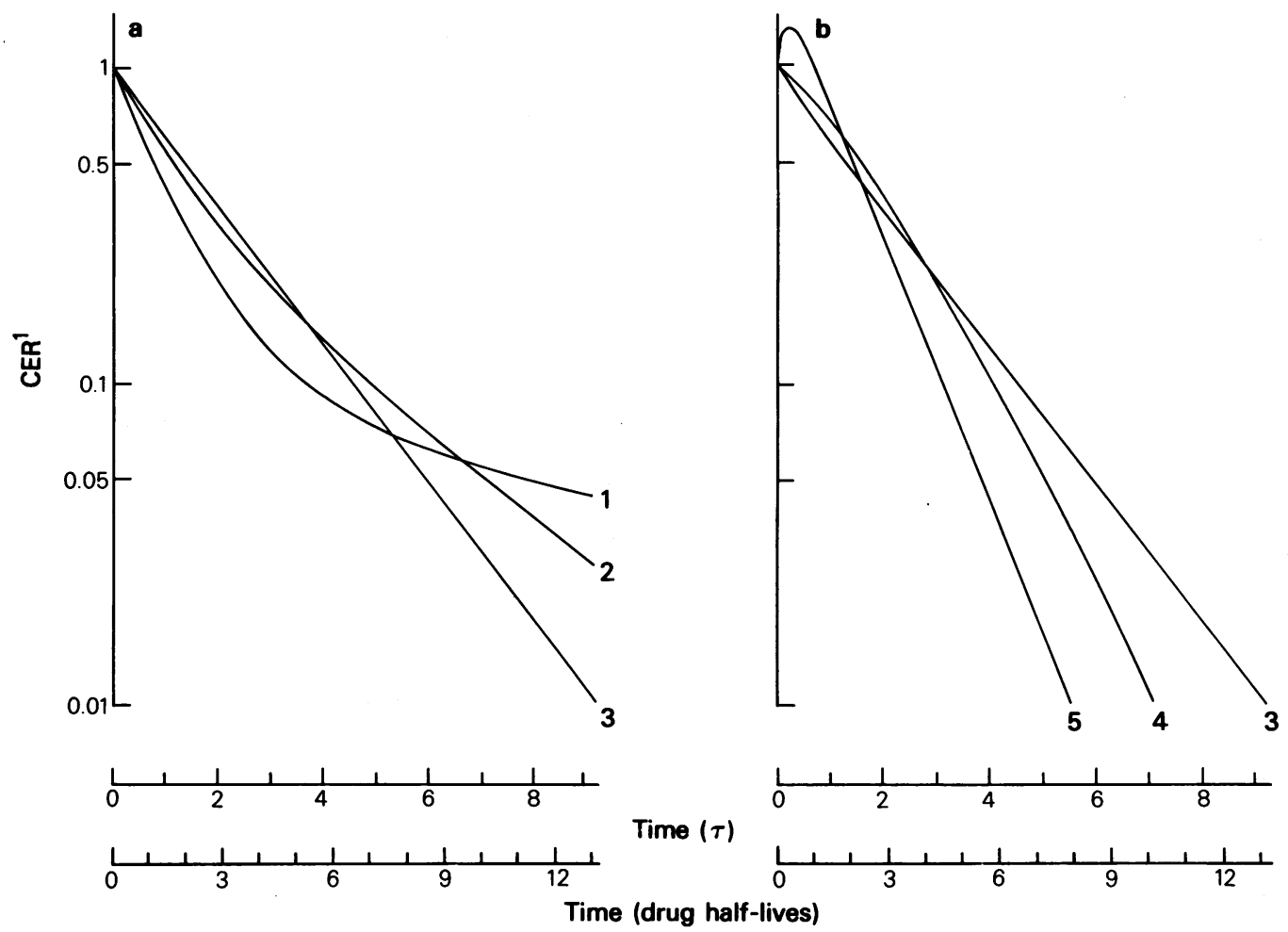

Figure $1{ }^{14} \mathrm{CO}_{2}$ exhalation rate-time profiles simulated using equation (9) for different ratios of $\mathrm{k} 2 / \mathrm{k} 1$. Curve 1 $(0.1), 2(0.3), 3(0.5), 4(0.8)$ and $5(5)$. CER 1 is the normalised ${ }^{14} \mathrm{CO}_{2}$ exhalation rate and the time scale is expressed in terms of $\tau(\tau=\mathrm{k} 1 . \mathrm{t})$ and AP half-lives $\left(t_{1 / 2}=0.693 / \mathrm{k} 1\right)$.

Table 1 Percentage error in initial and terminal rate constants estimated by curve stripping of simulated data obtained over different experimental time periods.

\begin{tabular}{|c|c|c|c|c|c|c|c|c|}
\hline \multirow{3}{*}{$\boldsymbol{K}$} & \multicolumn{4}{|c|}{$\begin{array}{l}\text { Percentage error in } \\
\text { initial rate constant }\end{array}$} & \multicolumn{4}{|c|}{$\begin{array}{l}\text { Percentage error in } \\
\text { terminal rate constant }\end{array}$} \\
\hline & \multicolumn{4}{|c|}{ Experimental time $e^{*}$} & \multicolumn{4}{|c|}{ Experimental time $e^{*}$} \\
\hline & 2 & 5 & 7 & 10 & 2 & 5 & 7 & 10 \\
\hline 0.1 & 94 & 13 & 4 & 1 & 900 & 100 & 21 & 2 \\
\hline 0.2 & 98 & 15 & 5 & 1 & 170 & 28 & 7 & 1 \\
\hline 0.3 & 93 & 18 & 7 & 2 & 62 & 12 & 3 & $<1$ \\
\hline 0.4 & 86 & 21 & 9 & 3 & 20 & 4 & 2 & $<1$ \\
\hline 0.45 & 97 & 23 & 10 & 4 & 9 & 2 & 1 & $<1$ \\
\hline
\end{tabular}

* Expressed as multiples of aminopyrine half-life. 
sufficient to enable the first term in equation (9) to approach zero. The influence of $T$ on the accuracy of estimates for the initial and terminal rate constants by curve stripping is shown in Table 1 . For a given $T$ the error associated with the terminal rate constant increases as $\mathrm{K}$ decreases, while the other rate constant is independent of $K$. The smaller $T$ the greater the error in both rate constants. The minimum $\mathrm{T}$ required to obtain rate constants with less than $4 \%$ error will be $10 \tau$ for the range of $K$ between 0.1 and 0.45 . This is equivalent to 14 half-lives of AP. It should be stressed that the results in Table 1 were generated with no experimental noise.

$K$ values higher than the critical value, where monoexponential behaviour is observed, result in convex CER-time profiles. The terminal phase of which may reflect either $\mathrm{k} 1$ or $\mathrm{k} 2$. In theory the other rate constant may be obtained by curve stripping. In the experimental situation an accrual phase is always evident in CER-time profiles regardless of $K$. This may be due to a number of factors including AP absorption (the oral route is frequently used) and the finite time necessary for the conversion of formaldehyde to $\mathrm{CO}_{2}$. Hence it is likely that only the terminal rate constant may be estimated in this situation, curve stripping being of limited use.

To summarise, the CER-time profiles simulated using the proposed model have demonstrated the complexity of the relationship betwen CER and demethylation of AP and MAP. When demethylation of AP and MAP is complete, a concave biexponential decline in CER will occur when the halflife ratio of MAP to AP exceeds approximately twofold $(\mathrm{K}<0.5)$. Incomplete demethylation will lower this critical half-life ratio. Unfortunately there are insufficient experimental data to validate the proposed model.

To date the AP-CER method has been studied more extensively in the rat than in other animal species. CER studies in 50 rats (Lockwood \&
Houston, unpublished data) revealed biexponential behaviour in approximately $80 \%$ of these animals with an average half-life ratio of 2 . However a biexponential decline in CER can be discerned in rats where the MAP/AP half-life ratio is 1.7 (Houston \& Lockwood, 1979). Thus either demethylation or subsequent oxidation of the single carbon fragment to $\mathrm{CO}_{2}$ is incomplete. Previous mass balance calculations (Lockwood \& Houston, 1979) using ${ }^{14} \mathrm{CO}_{2}$ exhalation and urinary demethylation product data, have shown that demethylation accounts for $80 \%$ of the elimination of AP, and $80 \%$ of the formaldehyde generated is ultimately recovered as ${ }^{14} \mathrm{CO}_{2}$. Thus the cumulative ${ }^{14} \mathrm{CO}_{2}$ exhaled following AP administration to rats is approximately $60 \%$ of dose. Limited studies in guinea pigs (Roots et al., 1980) would suggest the MAP/AP half-life ratio is less in this species. However, our simulations would suggest that in this study the experimental time was insufficient to obtain accurate estimates of AP and MAP.

The situation in man has yet to be investigated adequately. The inequivalence of plasma and CER half-lives reported (Bircher et al., 1976; Noordhoek et al., 1978) would suggest that the latter half-life is a hybrid parameter as predicted from our model. However human CER-time profiles (Bircher et al., 1976; Hepner \& Vesell, 1976; Noordhoek et al., 1978; Henry et al., 1979) have only been investigated over a 6-8 $\mathrm{h}$ time period (equivalent to $3-4$ times the plasma half-life of AP). The cumulative recovery of ${ }^{14} \mathrm{CO}_{2}$ following AP administration to man is approximately $25 \%$ of dose (Gikalov \& Bircher, 1977; Henry et al., 1979). In order to determine CER over an adequate time period to allow resolution of the two phases (at least $24 \mathrm{~h}$ ) the $2 \mu \mathrm{Ci}$ tracer dose commonly administered would need to be increased to $10 \mu \mathrm{Ci}$ or possibly more.

JCR is grateful to the Science Research Council for financial support.

\section{References}

AEBI, H., QUITT, J. \& LAUBER, E. (1963). Demethylierung in vivo und in vitro von Methixen. Naunyn-Schmiedebergs Arch. Pharmac., 244, 477-492.

BIRCHER, J., KUPFER, A., GIKALOV, I. \& PREISIG, R. (1976). Aminopyrine demethylation measured by breath analysis. Clin. Pharmac. Ther., 20, 484-492.

BRODIE, B.B \& AXELROD, J. (1950). Fate of aminopyrine in man and methods for estimation of aminopyrine and its metabolites in biological material. J. Pharmac. exp. Ther., 99, 171-184:

GIBALDI, M. \& PERRIER, D. (1975). Drugs and Pharmaceutical Sciences Vol. 1. Pharmacokinetics, pp. 281-292. New York: Marcel Dekker.
GIKALOV, I. \& BIRCHER, J. (1977). Dose dependence of the aminopyrine breath test. Eur. J. clin. Pharmac., 12, 229-233.

HENRY, D.A., SHARPE, G., CHAPLAIN, S., CARTWRIGHT, S., KITCHINGMAN, G., BELL, G.D. \& LANGMAN, M.J.S. (1979). [ [ $\left.{ }^{14} \mathrm{C}\right]$-Aminopyrine breath test. Comparison of different forms of analysis. Br. J. clin. Pharmac., 8, 539-545.

HEPNER, G.W. \& VESELL, E. (1975). Quantitative assessment of hepatic function by breath analysis after oral administraiton of $\left[{ }^{14} \mathrm{C}\right]$-aminopyrine. Ann. Int. Med., 83, 632-638.

HEPNER, G.W. \& VESELL, E. (1976). Aminopyrine disposi- 
tion: studies on breath, saliva and urine of normal subjects and patients with liver disease. Clin. Pharmac. Ther., 20, 654-661.

HEPNER, G.W., VESELL, E. TANTUM, K.R. (1978). Reduced drug elimination in congestive heart failure. Studies using aminopyrine as a model drug. Am. J. Med., 65, 271-276.

HOUSTON, J.B. \& LOCKWOOD, G.F. (1979). Aminopyrine demethylation kinetics: comparison of plasma exhaled metabolites. Br. J. Pharmac., 66, 429P.

HOUSTON, J.B., LOCKWOOD, G.F. \& TAYLOR, G. (1981). Aminopyrine demethylation kinetics. Use of metabolite exhalation rates as an index of enhanced mixed function oxidase activity in vivo. Drug Metab. Dispos., 9, $449-455$

LAUTERBURG, B. \& BIRCHER, J. (1973). Hepatic microsomal drug metabolising capacity measured in vivo by breath analysis. Gastroenterology, 65, A-32/556.

LOCKWOOD, G.F. \& HOUSTON, J.B. (1979). Amidopyrine disposition in rat. J. Pharm. Pharmac., 31, 787-788.

LOCKWOOD, G.F. \& HOUSTON, J.B. (1980). Influence of phenobarbitone pretreatment on disposition of amidopyrine and its metabolites in rat. J. Pharm. Pharmac., 32, 619-623.

NOORDHOEK, J., DEES, J., SAVENIJE-CHAPEL, E.M. \& WILSON, J.H.P. (1978). Output of ${ }^{1+} \mathrm{CO}_{2}$ in breath after oral administration of $\left({ }^{14} \mathrm{C}\right.$-methyl)-aminopyrine in hepatitis cirrhosis and hepatic bilharziasis: its relationships to aminopyrine pharmacokinetics. Eur. J. clin. Pharmac., 131, 223-229.

ROOTS, I., LEY, B., SETIAWAN, L., HEINEMEYER, G. \& HILDEBRANDT, A.G. (1978). Spironolactone. Characterisation of its enzyme-inducing properties by in vivo parameters. In Aldosterone antagonists in clinical medicine, eds Addison, G.M., Wirenfeldt Asmussen, N., Corvol, P., Kloppenborg, P.W.C., Norman, N., Schroder, R. \& Robertson, J.I.S., pp. 165-176. Amsterdam: Excerpta Medica.

ROOTS, I., NIGAM, S., GRAMATZKI, S., HEINEMYER, G. \& HILDEBRANDT, A.G. (1980). Hybrid information provided by ${ }^{14} \mathrm{C}$-aminopyrine breath test. Studies with ${ }^{14} \mathrm{C}$ monomethylaminoantipyrine in the guinea pig. Naunyn-Schmiedebergs Arch. Pharmac., 313, 175-178.

WAYDHAS, C., WEIGL, K. \& SIES, H. (1978). Disposition of formaldehyde and formate arising from drug $\mathrm{N}$ demethylations dependent on cytochrome $\mathrm{P}-45 \mathrm{O}$ in hepatocytes and in perfused rat liver. Eur. J. Biochem., 89, 143-150. 